\title{
SKEW COMPRESSIONS OF POSITIVE DEFINITE OPERATORS AND MATRICES*
}

\author{
MATTEO POLETTINI ${ }^{\dagger}$ AND ALBRECHT BÖTTCHER
}

\begin{abstract}
The paper is devoted to results connecting the eigenvalues and singular values of operators composed by $P^{*} G P$ with those composed in the same way by $Q G^{-1} Q^{*}$. Here $P$ and $Q$ are skew complementary projections on a finite-dimensional Hilbert space and $G$ is a positive definite linear operator on this space. Also discussed are graph theoretic interpretations of one of the results.
\end{abstract}

Key words. Skew projections, Eigenvalues, Singular values, Spanning-tree polynomials.

AMS subject classifications. 15A18, 05C $31,15 \mathrm{~A} 15,15 \mathrm{~B} 57$.

1. Introduction and main results. Let $\mathcal{H}$ be a real or complex $n$-dimensional Hilbert space, let $G \in \mathcal{L}(\mathcal{H})$ be a (selfadjoint and) positive definite operator, and let $P \neq 0, I$ be a projection on $\mathcal{H}$, that is, an operator in $\mathcal{L}(\mathcal{H})$ satisfying $P^{2}=P$. We denote by $Q=I-P$ the complementary projection. The metrics induced by $G$ on the ranges $\operatorname{Im} P$ and $\operatorname{Im} Q$ are given by $(G P x, P y)=\left(P^{*} G P x, y\right)$ and $(G Q x, Q y)=$ $\left(Q^{*} G Q x, y\right)$. Analogously, the metrics generated by $G^{-1}$ on $\operatorname{Im} P^{*}$ and $\operatorname{Im} Q^{*}$ lead to the operators $P G^{-1} P^{*}$ and $Q G^{-1} Q^{*}$. This paper is about some interesting connections between the metrics induced by $G$ and $G^{-1}$ on the oblique subspaces.

The four operators $P^{*} G P, P G^{-1} P^{*}, Q G^{-1} Q^{*}, Q^{*} G Q$ are not invertible, and hence, their determinants are zero. Given an operator $A \in \mathcal{L}(\mathcal{H})$, we denote by $\operatorname{det}_{+} A$ the product of the nonzero roots of the characteristic polynomial $\operatorname{det}(\lambda I-A)$ taken with their multiplicity. As the operators $P^{*} G P, P G^{-1} P^{*}, Q G^{-1} Q^{*}, Q^{*} G Q$ are selfadjoint and positive semi-definite, their eigenvalues coincide with the zeros of their characteristic polynomials and are nonnegative real numbers. Thus, det+ of these operators is simply the product of the nonzero eigenvalues. Geometrically, if $\mathcal{H}$ is a real space, then $\operatorname{det}_{+}\left(P^{*} G P\right)$ is the volume element in the metric on $\operatorname{Im} P$ induced by $G$, that is, the volume of $P(V) \subset \operatorname{Im} P$ where $V \subset \mathcal{H}$ is the cube spanned by any orthonormal basis in $\mathcal{H}$. Here is our first result.

THEOREM 1.1. We have

$$
\operatorname{det} G=\frac{\operatorname{det}_{+}\left(P^{*} G P\right)}{\operatorname{det}_{+}\left(Q G^{-1} Q^{*}\right)}=\frac{\operatorname{det}_{+}\left(Q^{*} G Q\right)}{\operatorname{det}_{+}\left(P G^{-1} P^{*}\right)}
$$

We remark that the first equality in the theorem remains true if the hypothesis that $G$ be positive definite is replaced by the sole requirement that $G$ and $P^{*} G P: \operatorname{Im} P \rightarrow \operatorname{Im} P^{*}$ be invertible; the invertibility of $Q G^{-1} Q^{*}: \operatorname{Im} Q^{*} \rightarrow \operatorname{Im} Q$ then follows automatically.

Theorem 1.1 implies that $\operatorname{det}_{+}\left(P^{*} G P\right) \operatorname{det}_{+}\left(P G^{-1} P^{*}\right)=\operatorname{det}_{+}\left(Q G^{-1} Q^{*}\right) \operatorname{det}_{+}\left(Q^{*} G Q\right)$. The map send-

\footnotetext{
*Received by the editors on April 7, 2020. Accepted for publication on April 30, 2020. Handling Editor: Ilya Spitkovsky. Corresponding Author: M. Polettini.

†Université du Luxembourg, Faculté des Sciences, de la Technologie et de la Communication, 162 A Avenue de la Faïencerie, L-1511 Luxembourg, Grand Duchy of Luxembourg (matteo.polettini@uni.lu).

‡Technische Universität Chemnitz, Fakultät für Mathematik, D-09107 Chemnitz, Germany (aboettch@mathematik.tuchemnitz.de).
} 
ing $A$ to $\operatorname{det}_{+} A$ is not multiplicative, not even on the special operators/matrices we are considering. Indeed, if we take $\mathcal{H}=\mathbb{R}^{2}, P=\left[\begin{array}{ll}1 & \omega \\ 0 & 0\end{array}\right]$, and $G=\left[\begin{array}{ll}a & 0 \\ 0 & b\end{array}\right]$, then

$$
\begin{aligned}
& \operatorname{det}_{+}\left(P^{*} G P\right)=a\left(1+\omega^{2}\right), \quad \operatorname{det}_{+}\left(P G^{-1} P^{*}\right)=\frac{1}{a}+\frac{1}{b} \omega^{2}, \\
& \operatorname{det}_{+}\left(Q G^{-1} Q^{*}\right)=\frac{1}{b}\left(1+\omega^{2}\right), \quad \operatorname{det}_{+}\left(Q^{*} G Q\right)=b+a \omega^{2},
\end{aligned}
$$

implying that

$$
\operatorname{det}_{+}\left(P^{*} G P\right) \operatorname{det}_{+}\left(P G^{-1} P^{*}\right)=\operatorname{det}_{+}\left(Q G^{-1} Q^{*}\right) \operatorname{det}_{+}\left(Q^{*} G Q\right)=\left(1+\frac{a}{b} \omega^{2}\right)\left(1+\omega^{2}\right),
$$

whereas

$$
\operatorname{det}_{+}\left(P^{*} G P G^{-1} P^{*}\right)=\operatorname{det}_{+}\left(Q G^{-1} Q^{*} G Q\right)=1+\frac{a}{b} \omega^{2} .
$$

However, this example suggests that the equality $\operatorname{det}_{+}\left(P^{*} G P G^{-1} P^{*}\right)=\operatorname{det}_{+}\left(Q G^{-1} Q^{*} G Q\right)$ might be true in general. Our second result shows that this is indeed the case. Moreover, it reveals that, except for the eigenvalue 0 and the possible eigenvalue 1 , the eigenvalues and their algebraic multiplicities of the operators $P^{*} G P G^{-1} P^{*}$ and $Q G^{-1} Q^{*} G Q$ coincide.

TheOREM 1.2. The spectra of the operators $P^{*} G P G^{-1} P^{*}$ and $Q G^{-1} Q^{*} G Q$ are contained in $\{0\} \cup[1, \infty)$, and a number $\lambda>1$ is an eigenvalue of algebraic multiplicity $\alpha$ of the first operator if and only if it is an eigenvalue of the same algebraic multiplicity of the second operator. In particular, $\operatorname{det}_{+}\left(P^{*} G P G^{-1} P^{*}\right)=$ $\operatorname{det}_{+}\left(Q G^{-1} Q^{*} G Q\right)$.

We will show below that the singular values of $P^{*} G P G^{-1} P^{*}$ and $Q G^{-1} Q^{*} G Q$ lying in $(1, \infty)$ need not coincide. However, we will prove that this happens if $G=I$, that is, except for the multiplicities of the singular values 0 and 1 , the operators $P^{*} P P^{*}$ and $Q Q^{*} Q$ have the same singular values with the same multiplicities. This can be generalized to the operators $f\left(P, P^{*}\right)$ and $f\left(Q^{*}, Q\right)$ where $f(x, y)$ is a polynomial in two non-commuting variables $x$ and $y$. Before doing this, we consider an example. Let

$$
f(x, y)=a+b x+c x y+d x^{2} y+e y x y
$$

with coefficients from the scalar field $\mathbb{K} \in\{\mathbb{R}, \mathbb{C}\}$, take $\mathcal{H}=\mathbb{K}^{3}$ with the usual scalar product, and let

$$
P=\left[\begin{array}{lll}
1 & s & 0 \\
0 & 0 & 0 \\
0 & 0 & 0
\end{array}\right], \quad P^{*}=\left[\begin{array}{lll}
1 & 0 & 0 \\
s & 0 & 0 \\
0 & 0 & 0
\end{array}\right], \quad Q=\left[\begin{array}{ccc}
0 & -s & 0 \\
0 & 1 & 0 \\
0 & 0 & 1
\end{array}\right], \quad Q^{*}=\left[\begin{array}{ccc}
0 & 0 & 0 \\
-s & 1 & 0 \\
0 & 0 & 1
\end{array}\right]
$$

with a real number $s$. We get

$$
\begin{aligned}
f\left(P, P^{*}\right) & =a I+b P+c P P^{*}+d P^{2} P^{*}+e P^{*} P P^{*}=a I+b P+(c+d) P P^{*}+e P^{*} P P^{*} \\
& =\left[\begin{array}{ccc}
a+b+(c+d+e)\left(1+s^{2}\right) & b s & 0 \\
e s\left(1+s^{2}\right) & a & 0 \\
0 & 0 & a
\end{array}\right], \\
f\left(Q^{*}, Q\right) & =a I+b Q^{*}+c Q^{*} Q+d\left(Q^{*}\right)^{2} Q+e Q Q^{*} Q=a I+b Q^{*}+(c+d) Q^{*} Q+e Q Q^{*} Q \\
& =\left[\begin{array}{ccc}
a & -e s\left(1+s^{2}\right) & 0 \\
-b s & a+b+(c+d+e)\left(1+s^{2}\right) & 0 \\
0 & 0 & a+b+c+d+e
\end{array}\right] .
\end{aligned}
$$


Let $\lambda_{1}, \lambda_{2}$ be the roots of the characteristic polynomial of the upper-left $2 \times 2$ block of $f\left(P, P^{*}\right)$. The lower-right entries of $f\left(P, P^{*}\right)$ and $f\left(Q^{*}, Q\right)$ are $f(0,0)$ and $f(1,1)$, respectively. Thus, the roots of the characteristic polynomials of $f\left(P, P^{*}\right)$ and $f\left(Q^{*}, Q\right)$ are

$$
\lambda_{1}, \lambda_{2}, f(0,0) \quad \text { and } \quad \lambda_{1}, \lambda_{2}, f(1,1),
$$

respectively. It follows that, up to $f(0,0)$ and $f(1,1)$, the operators $f\left(P, P^{*}\right)$ and $f\left(Q^{*}, Q\right)$ have the same eigenvalues with the same algebraic multiplicities. Computing $f\left(P, P^{*}\right)\left[f\left(P, P^{*}\right)\right]^{*}$ and $f\left(Q^{*}, Q\right)\left[f\left(Q^{*}, Q\right)\right]^{*}$ one will see that, this time up to $|f(0,0)|$ and $|f(1,1)|$, the operators $f\left(P, P^{*}\right)$ and $f\left(Q^{*}, Q\right)$ have the same singular values with the same multiplicities. Herewith our third result. Recall that $\mathbb{K} \in\{\mathbb{R}, \mathbb{C}\}$ stands for the scalar field of $\mathcal{H}$.

THEOREM 1.3. Let $f(x, y)$ be a polynomial in two non-commuting variables $x, y$ with coefficients from the scalar field $\mathbb{K}$. A number $\lambda \in \mathbb{K} \backslash\{f(0,0), f(1,1)\}$ is an eigenvalue of algebraic multiplicity $\alpha$ of $f\left(P, P^{*}\right)$ if and only if it is an eigenvalue of the same algebraic multiplicity of $f\left(Q^{*}, Q\right)$, and a nonnegative number $s \notin\{|f(0,0)|,|f(1,1)|\}$ is a singular value of multiplicity $\alpha$ of $f\left(P, P^{*}\right)$ if and only if it is a singular value of the same multiplicity of $f\left(Q^{*}, Q\right)$. If $f(x, y)$ is a monomial, then the singular values of $f\left(P, P^{*}\right)$ and $f\left(Q^{*}, Q\right)$ belong to $\{0\} \cup[1, \infty)$ and $\left\|f\left(P, P^{*}\right)\right\|=\left\|f\left(Q^{*}, Q\right)\right\|$.

If $f(x, y)=x$, we get the classical equality $\|P\|=\left\|Q^{*}\right\|$, which implies that actually $\|P\|=\left\|P^{*}\right\|=$ $\|Q\|=\left\|Q^{*}\right\|$; see [1] and especially [9] for the history and for several proofs of this equality. For higher-degree monomials $f(x, y)$, we may restrict ourselves to the cases where $f(x, y)=x y x \cdots$ or $f(x, y)=y x y \cdots$ with $m \geq 2$ factors. (Note that, for example, $P^{2}\left(P^{*}\right)^{3} P^{6}=P P^{*} P$.) Thus, if $P=U S V$ is the singular value decomposition of $P$, then $f\left(P, P^{*}\right)=P P^{*} P \cdots=U S^{m} V$ or $f\left(P, P^{*}\right)=P^{*} P P^{*} \cdots=V^{*} S^{m} U^{*}$. It follows that the singular values of $f\left(P, P^{*}\right)$ are just the $m$ th powers of the singular values of $P$. In particular, $\left\|f\left(P, P^{*}\right)\right\|=\|P\|^{m}$, and as we already know that $\|P\|=\|Q\|$, we arrive at the conclusion that

$$
\|\underbrace{P P^{*} P \cdots}_{m}\|=\|\underbrace{P^{*} P P^{*} \cdots}_{m}\|=\|\underbrace{Q Q^{*} Q \cdots}_{m}\|=\|\underbrace{Q^{*} Q Q^{*} \cdots}_{m}\|=\|P\|^{m}=\|Q\|^{m} .
$$

What about the norm equality if $f(x, y)$ is not a monomial? Feldman, Krupnik, and Markus [3] showed that if $\operatorname{dim} \mathcal{H}=2$, then $\left\|f\left(P, P^{*}\right)\right\|=\psi(\|P\|)$ with some function $\psi(x)$. Thus, in this case, $\left\|f\left(P, P^{*}\right)\right\|$ depends solely on $\|P\|$ and we conclude that $\left\|f\left(P, P^{*}\right)\right\|=\left\|f\left(Q^{*}, Q\right)\right\|$ for every polynomial $f(x, y)$. A theorem by Spigel [8] says that if $P, R$ are two nontrivial projections in $\mathcal{L}(\mathcal{H})$, then the equality $\left\|f\left(P, P^{*}\right)\right\|=$ $\left\|f\left(R, R^{*}\right)\right\|$ is true for all polynomials $f(x, y)$ if and only if

$$
\sigma\left(P P^{*} P\right) \backslash\{0,1\}=\sigma\left(R R^{*} R\right) \backslash\{0,1\}
$$

and

$$
\sigma\left(P+2 P^{*}\right) \cap\{0,1,2,3\}=\sigma\left(R+2 R^{*}\right) \cap\{0,1,2,3\},
$$

where $\sigma(A)$ stands for the spectrum of $A$ (= set of the zeros of $\operatorname{det}(\lambda I-A)$ ). Now let $P$ be an arbitrary nontrivial projection and take $R=Q^{*}$ with $Q=I-P$. Then equality (1.3) is always satisfied due to Theorem 1.2. However, if we take $P$ as in (1.1) with $s=1$, then the eigenvalues of

$$
P+2 P^{*}=\left[\begin{array}{lll}
3 & 1 & 0 \\
2 & 0 & 0 \\
0 & 0 & 0
\end{array}\right] \text { and } R+2 R^{*}=Q^{*}+2 Q=\left[\begin{array}{ccc}
0 & -2 & 0 \\
-1 & 3 & 0 \\
0 & 0 & 3
\end{array}\right]
$$


are $0,(3 \pm \sqrt{17}) / 2$ and $3,(3 \pm \sqrt{17}) / 2$, respectively, so that (1.4) is not satisfied. Thus, the equality $\left\|f\left(P, P^{*}\right)\right\|=\left\|f\left(Q^{*}, Q\right)\right\|$ cannot be true for all polynomials $f(x, y)$. We will show that, in the general case, $\left\|f\left(P, P^{*}\right)\right\|=\left\|f\left(Q^{*}, Q\right)\right\|$ for all polynomials $f(x, y)$ if and only if

$$
\sigma\left(P+2 P^{*}\right) \cap\{0\}=\sigma\left(Q^{*}+2 Q\right) \cap\{0\} .
$$

We remark that Theorems 1.1 and 1.2 were established in [6]. We here give proofs that are significantly shorter than those in [6]. Theorem 1.3 is in the vein of Feldman, Krupnik, Markus [3] and Spigel [8], but in the form stated and proved here it cannot be found in these two papers. Our proof makes use of the Doković canonical form of skew projections, for which see [2] or [1, Corollary 2.3]. Finally, following [5] and [6], we embark on the connection of Theorem 1.1 with graph theory. We will not provide new graph theoretic results, we rather want to point out that this theorem admits nice graph theoretic interpretations and that it has been implicit in the graph theoretic literature for a long time.

2. Proofs. Choose orthonormal bases in $\operatorname{Im} P$ and $\operatorname{Im} Q^{*}=(\operatorname{Im} P)^{\perp}$. The union of these two bases is an orthonormal basis in $\mathcal{H}$. The matrix representations of $P, P^{*}, Q, Q^{*}$ in this basis are

$$
P=\left[\begin{array}{ll}
I & \omega \\
0 & 0
\end{array}\right], \quad P^{*}=\left[\begin{array}{cc}
I & 0 \\
\omega^{*} & 0
\end{array}\right], \quad Q=\left[\begin{array}{cc}
0 & -\omega \\
0 & I
\end{array}\right], \quad Q^{*}=\left[\begin{array}{cc}
0 & 0 \\
-\omega^{*} & I
\end{array}\right]
$$

Let $k$ be the dimension of $\operatorname{Im} P$. Then $1 \leq k \leq n-1$, and $\ell=n-k$ is the dimension of $\operatorname{Im} Q^{*}$. By symmetry, we may without loss of generality suppose that $k \leq \ell$. Clearly, $\omega$ is a $k \times \ell$ matrix.

The representations (2.6) were first employed by Feldman, Krupnik, and Markus in [3] and were independently used in [9, page 312], where they are cited as a private communication by Gustavo Corach. They are also as Corollary 1.7 in [1]; this corollary is stated for complex spaces, but it holds verbatim also for real spaces.

Proof of Theorem 1.1. By symmetry, it is enough to prove the first equality. Let $G=\left[\begin{array}{ll}A & B \\ C & D\end{array}\right]$ be the matrix representation of $G$ in our orthonormal basis and let $G^{-1}=\left[\begin{array}{ll}a & b \\ c & d\end{array}\right]$. We obtain

$$
P^{*} G P=\left[\begin{array}{cc}
A & A \omega \\
\omega^{*} A & \omega^{*} A \omega
\end{array}\right], \quad Q G^{-1} Q^{*}=\left[\begin{array}{cc}
\omega d \omega^{*} & -\omega d \\
-d \omega^{*} & d
\end{array}\right]
$$

The identities

$$
\left[\begin{array}{cc}
A & A \omega \\
\omega^{*} A & \omega^{*} A \omega
\end{array}\right]=\left[\begin{array}{cc}
I & -\omega \\
\omega^{*} & I
\end{array}\right]\left[\begin{array}{cc}
A\left(I+\omega \omega^{*}\right) & 0 \\
0 & 0
\end{array}\right]\left[\begin{array}{cc}
I & -\omega \\
\omega^{*} & I
\end{array}\right]^{-1}
$$

and

$$
\left[\begin{array}{cc}
\omega d \omega^{*} & -\omega d \\
-d \omega^{*} & d
\end{array}\right]=\left[\begin{array}{cc}
I & -\omega \\
\omega^{*} & I
\end{array}\right]\left[\begin{array}{cc}
0 & 0 \\
0 & d\left(I+\omega^{*} \omega\right)
\end{array}\right]\left[\begin{array}{cc}
I & -\omega \\
\omega^{*} & I
\end{array}\right]^{-1}
$$

can be verified straightforwardly. They tell us that $P^{*} G P$ and $Q G^{-1} Q^{*}$ have the same eigenvalues as the block-diagonal matrices on the right. Consequently,

$$
\operatorname{det}_{+}\left(P^{*} G P\right)=\operatorname{det} A \operatorname{det}\left(I+\omega \omega^{*}\right), \quad \operatorname{det}_{+}\left(Q G^{-1} Q^{*}\right)=\operatorname{det} d \operatorname{det}\left(I+\omega^{*} \omega\right) .
$$

It follows that

$$
\frac{\operatorname{det}_{+}\left(P^{*} G P\right)}{\operatorname{det}_{+}\left(Q G^{-1} Q^{*}\right)}=\frac{\operatorname{det} A \operatorname{det}\left(I+\omega \omega^{*}\right)}{\operatorname{det} d \operatorname{det}\left(I+\omega^{*} \omega\right)}
$$


and Jacobi's determinant identity $\operatorname{det} d=(1 / \operatorname{det} G) \operatorname{det} A$ and the easily verified $\operatorname{identity} \operatorname{det}\left(I+\omega \omega^{*}\right)=$ $\operatorname{det}\left(I+\omega^{*} \omega\right)$ complete the proof.

Our proof of Theorem 1.2 makes use of the following known facts. First, let $X_{1}$ and $X_{2}$ be linear spaces, $A: X_{1} \rightarrow X_{2}$ be a linear and invertible operator, and $P_{1}: X_{1} \rightarrow X_{1}$ and $P_{2}: X_{2} \rightarrow X_{2}$ be linear projections. Put $Q_{1}=I-P_{1}$ and $Q_{2}=I-P_{2}$. Then $P_{2} A P_{1}: \operatorname{Im} P_{1} \rightarrow \operatorname{Im} P_{2}$ is invertible if and only if $Q_{1} A^{-1} Q_{2}: \operatorname{Im} Q_{2} \rightarrow \operatorname{Im} Q_{1}$ is invertible. In that case,

$$
P_{1}\left(P_{2} A P_{1}\right)^{-1} P_{2}=P_{1} A^{-1} P_{2}-P_{1} A^{-1} Q_{2}\left(Q_{1} A^{-1} Q_{2}\right)^{-1} Q_{1} A^{-1} P_{2} .
$$

Second, denote by $\sigma(C)$ the spectrum of $C \in \mathcal{L}(\mathcal{H})$ as a multiset, that is, each point is repeated according to its algebraic multiplicity. It is well known that if $A$ is a $k \times \ell$ matrix and $B$ is an $\ell \times k$ matrix with $k \leq \ell$, then the characteristic polynomials of $A B$ and $B A$ are related by the equality

$$
\operatorname{det}(\lambda I-B A)=\lambda^{\ell-k} \operatorname{det}(\lambda I-A B)
$$

Thus, the well known equality

$$
\sigma(A B) \backslash\{0,0, \ldots\}=\sigma(B A) \backslash\{0,0, \ldots\}
$$

is true as an equality for multisets. Third, if $R=\left[\begin{array}{ll}I & \omega \\ 0 & 0\end{array}\right]$ is a projection and $A=\left[\begin{array}{ll}B & C \\ D & E\end{array}\right]$ an equally partitioned matrix, then

$$
I-R A R=\left[\begin{array}{cc}
I-B-\omega D & * \\
0 & I
\end{array}\right], \quad R-R A R=\left[\begin{array}{cc}
I-B-\omega D & * \\
0 & 0
\end{array}\right]
$$

which shows that

$$
\sigma(R-R A R) \backslash\{0,0, \ldots, 1,1, \ldots\}=\sigma(I-R A R) \backslash\{0,0, \ldots, 1,1, \ldots\},
$$

again as an equality for multisets. Finally, we also have $\sigma(I-A)=1-\sigma(A)$ as an equality for multisets. We write $S \cong T$ for two multisubsets of $\mathbb{C}$ if $S \backslash\{0,0, \ldots, 1,1, \ldots\}=T \backslash\{0,0, \ldots, 1,1, \ldots\}$ as multisets.

Proof of Theorem 1.2. Replacing $Q^{*}$ in $Q G^{-1} Q^{*} G Q$ by $I-P^{*}$, we get $Q-Q G^{-1} P^{*} G Q$. Making such a replacement twice and taking into account (2.8) and (2.9) we obtain

$$
\begin{aligned}
& \sigma\left(Q G^{-1} Q^{*} G Q\right)=\sigma\left(Q-Q G^{-1} P^{*} G Q\right) \cong \sigma\left(I-Q G^{-1} P^{*} G Q\right) \\
& =1-\sigma\left(Q G^{-1} P^{*} G Q\right) \cong 1-\sigma\left(P^{*} G Q G^{-1} P^{*}\right)=1-\sigma\left(P^{*}-P^{*} G P G^{-1} P^{*}\right) \\
& \cong 1-\sigma\left(I-P^{*} G P G^{-1} P^{*}\right)=1-\left(1-\sigma\left(P^{*} G P G^{-1} P^{*}\right)\right)=\sigma\left(P^{*} G P G^{-1} P^{*}\right),
\end{aligned}
$$

as asserted.

We are left with showing that $\sigma\left(P G^{-1} P^{*} G P\right) \subset\{0\} \cup[1, \infty)$. We know from the proof of Theorem 1.1 that $P G^{-1} P^{*}: \operatorname{Im} P^{*} \rightarrow \operatorname{Im} P$ is invertible, and from (2.7) we get

$$
P^{*} G P=\left(P G^{-1} P^{*}\right)^{-1} P+P^{*} G Q\left(Q^{*} G Q\right)^{-1} Q^{*} G P .
$$

Multiplying this equality by $P G^{-1} P^{*}$ from the left we obtain

$$
P G^{-1} P^{*} G P=P+P G^{-1} P^{*} G Q\left(Q^{*} G Q\right)^{-1} Q^{*} G P=: P+M .
$$

By $(2.9), \sigma\left(P G^{-1} P^{*} G P\right) \cong 1+\sigma(M)$. The operator

$$
L:=\left(P G^{-1} P^{*}\right)^{1 / 2} P^{*} G Q\left(Q^{*} G Q\right)^{-1} Q^{*} G P\left(P G^{-1} P^{*}\right)^{1 / 2}
$$


Electronic Journal of Linear Algebra, ISSN 1081-3810

A publication of the International Linear Algebra Society

Volume 36, pp. 400-410, June 2020.

is selfadjoint and positive, whence $\sigma(L) \subset[0, \infty)$, and from (2.8) we deduce that $\sigma(M) \cong \sigma(L)$. In summary $\sigma\left(P G^{-1} P^{*} G P\right) \cong 1+\sigma(L) \subset[1, \infty)$.

Counterexample. Theorem 1.2 cannot be transferred from eigenvalues to singular values. A counterexample is as follows. Let $\mathcal{H}=\mathbb{R}^{4}$, take $P, Q$ as in (2.6) with $\omega=\left[\begin{array}{ll}1 & 1 \\ 1 & 1\end{array}\right]$, and $\operatorname{choose} G=\operatorname{diag}[1,2,1,1]$. Straightforward computation gives

$$
P_{G}:=P^{*} G P G^{-1} P^{*}=\left[\begin{array}{cccc}
3 & 2 & 0 & 0 \\
4 & 5 & 0 & 0 \\
7 & 7 & 0 & 0 \\
7 & 7 & 0 & 0
\end{array}\right], \quad Q_{G}:=Q G^{-1} Q^{*} G Q=\left[\begin{array}{cccc}
0 & 0 & -7 & -7 \\
0 & 0 & -7 & -7 \\
0 & 0 & 4 & 3 \\
0 & 0 & 3 & 4
\end{array}\right] .
$$

Both matrices have the same eigenvalues $0,0,1,7$, which is in accordance with Theorem 1.2. Again by direct calculation,

$$
P_{G}^{*} P_{G}=\left[\begin{array}{cccc}
123 & 124 & 0 & 0 \\
124 & 127 & 0 & 0 \\
0 & 0 & 0 & 0 \\
0 & 0 & 0 & 0
\end{array}\right], \quad Q_{G}^{*} Q_{G}=\left[\begin{array}{cccc}
0 & 0 & 0 & 0 \\
0 & 0 & 0 & 0 \\
0 & 0 & 123 & 122 \\
0 & 0 & 122 & 123
\end{array}\right]
$$

The singular values of $P^{*} G P G^{-1} P^{*}$ and $Q G^{-1} Q^{*} G Q$ are the square roots of the eigenvalues of $P_{G}^{*} P_{G}$ and $Q_{G}^{*} Q_{G}$. But the latter two matrices cannot have the same eigenvalues because the traces of their nonzero $2 \times 2$ blocks are $250 \neq 246$. In fact, solving two quadratic equations we see that the singular values of $P_{G}=P^{*} G P G^{-1} P^{*}$ are $0,0,0.9919,15.7802$ and that those of $Q_{G}=Q G^{-1} Q^{*} G Q$ are $0,0,1,15.6525$.

Proof of Theorem 1.3. Doković [2] showed that if the scalar field is $\mathbb{C}$, then there is an orthonormal basis in which $P$ has a matrix of the form

$$
I_{r} \oplus O_{t} \oplus \operatorname{diag}\left\{\left[\begin{array}{cc}
1 & s_{j} \\
0 & 0
\end{array}\right]\right\}_{j=1}^{m}
$$

with real numbers $s_{j}>0$. The proof of [1, Corollary 2.3] reveals that this representation can also be achieved for the scalar field $\mathbb{R}$. Thus, in either case, there is a unitary operator $V$ such that $V^{*} f\left(P, P^{*}\right) V$ is given on $\mathbb{K}^{n}$ by

$$
f(1,1) I_{r} \oplus f(0,0) I_{t} \oplus \operatorname{diag}\left\{f\left(\left[\begin{array}{cc}
1 & s_{j} \\
0 & 0
\end{array}\right],\left[\begin{array}{cc}
1 & 0 \\
s_{j} & 0
\end{array}\right]\right)\right\}_{j=1}^{m}=: f(1,1) I_{r} \oplus f(0,0) I_{t} \oplus D_{P}
$$

It follows that $V^{*} f\left(Q^{*}, Q\right) V$ equals

$$
f(0,0) I_{r} \oplus f(1,1) I_{t} \oplus \operatorname{diag}\left\{f\left(\left[\begin{array}{cc}
0 & 0 \\
-s_{j} & 1
\end{array}\right],\left[\begin{array}{cc}
0 & -s_{j} \\
0 & 1
\end{array}\right]\right)\right\}_{j=1}^{m}=: f(0,0) I_{r} \oplus f(1,1) I_{t} \oplus D_{Q} .
$$

Thus, up to the possible eigenvalues $f(0,0)$ and $f(1,1)$, the roots of the characteristic polynomials of $f\left(P, P^{*}\right)$ and $f\left(Q^{*}, Q\right)$ are the roots of the characteristic polynomials of $D_{p}$ and $D_{Q}$, respectively. With the unitary matrix $U=\left[\begin{array}{cc}0 & 1 \\ -1 & 0\end{array}\right]$, we have

$$
\left[\begin{array}{cc}
0 & 0 \\
-s_{j} & 1
\end{array}\right]=U\left[\begin{array}{cc}
1 & s_{j} \\
0 & 0
\end{array}\right] U^{*}, \quad\left[\begin{array}{cc}
0 & -s_{j} \\
0 & 1
\end{array}\right]=U\left[\begin{array}{cc}
1 & 0 \\
s_{j} & 0
\end{array}\right] U^{*}
$$

which shows that $D_{P}$ and $D_{Q}$ are unitarily similar and hence have the same charcteristic polnomials. This completes the proof of the theorem for the eigenvalues. 
The squared singular values are the eigenvalues of $f\left(P, P^{*}\right)\left[f\left(P, P^{*}\right)\right]^{*}$ and $f\left(Q^{*}, Q\right)\left[f\left(Q^{*}, Q\right)\right]^{*}$. By straightforward computation we obtain that

$$
f\left(P, P^{*}\right)\left[f\left(P, P^{*}\right)\right]^{*}=g\left(P, P^{*}\right), \quad f\left(Q^{*}, Q\right)\left[f\left(Q^{*}, Q\right)\right]^{*}=g\left(Q^{*}, Q\right)
$$

with a polynomial $g(x, y)$ such that $g(0,0)=|f(0,0)|^{2}$ and $g(1,1)=|f(1,1)|^{2}$. (Example: If $f(x, y)=$ $a+b x+c x y$, then $g(x, y)=(a+b x+c x y)(\bar{a}+\bar{b} y+\bar{c} x y)$.) The assertion for the eigenvalues already proved therefore implies the assertion for the singular values.

We are left with the case where $f(x, y)$ is a monomial. By what was said after the statement of Theorem 1.3, it suffices to consider $f(x, y)=x$ only. However, the reasoning is equally simple in the general case. Namely, for the monomials $f(x, y)=x, x y, x y x, x y x y, x y x y x, \ldots$, we have $f(0,0)=0$ and $f(1,1)=1$, and if $P=\left[\begin{array}{ll}0 & s \\ 0 & 0\end{array}\right]$, then $f\left(P, P^{*}\right)$ takes the values

$$
\left[\begin{array}{ll}
1 & s \\
0 & 0
\end{array}\right],\left[\begin{array}{cc}
1+s^{2} & 0 \\
0 & 0
\end{array}\right],\left[\begin{array}{cc}
1+s^{2} & s\left(1+s^{2}\right) \\
0 & 0
\end{array}\right],\left[\begin{array}{cc}
\left(1+s^{2}\right)^{2} & 0 \\
0 & 0
\end{array}\right],\left[\begin{array}{cc}
\left(1+s^{2}\right)^{2} & s\left(1+s^{2}\right)^{2} \\
0 & 0
\end{array}\right], \ldots
$$

It is readily seen that the singular values of these matrices are 0 and $\left(1+s^{2}\right)^{\nu / 2}$ if the degree of $f(x, y)$ is $\nu$. Consequently, from the Doković representation and from what was already proved we infer that all singular values belong to $\{0\} \cup[1, \infty)$ and that the maximal singular value of $f\left(P, P^{*}\right)$, which is equal to the maximal singular value of $f\left(Q^{*}, Q\right)$, is strictly greater than 1 .

Proof of (1.5). This is easy with the Doković representation. We use Spigel's theorem cited in Section 1. (Note that there $\sigma(A)$ is understood as a true set and not as a multiset.) We know from Theorem 1.3 that (1.3) is satisfied for $P$ and $R=Q^{*}$. So we are left with showing that for $P$ and $R=Q^{*}$ the equalities (1.4) and (1.5) are equivalent. From (2.10) and (2.11) with $f(x, y)=x+2 y$ we infer that

$$
P+2 P^{*}=3 I_{r} \oplus 0_{t} \oplus \operatorname{diag}\left\{\left[\begin{array}{cc}
3 & s_{j} \\
2 s_{j} & 0
\end{array}\right]\right\}_{j=1}^{m}, \quad Q^{*}+2 Q=0_{r} \oplus 3 I_{t} \oplus \operatorname{diag}\left\{\left[\begin{array}{cc}
0 & -2 s_{j} \\
-s_{j} & 3
\end{array}\right]\right\}_{j=1}^{m} .
$$

The spectra of the diagonal blocks are the solutions of the equation $\lambda^{2}-3 \lambda-2 s_{j}^{2}=0$. This equation is never satisfied for $\lambda \in\{0,1,2,3\}$. Consequently, (1.4) holds if and only if either $r=t=0$ or $r t>0$. But the requirement $r=t=0$ is equivalent to the equalities $\sigma\left(P+2 P^{*}\right) \cap\{0\}=\sigma\left(Q^{*}+2 Q\right) \cap\{0\}=\emptyset$, while the inequality $r t>0$ is the same as saying that $\sigma\left(P+2 P^{*}\right) \cap\{0\}=\sigma\left(Q^{*}+2 Q\right) \cap\{0\}=\{0\}$.

3. The connection with graph theory. Theorem 1.1 has a graph theoretic interpretation. We confine ourselves to illustrate this connection by an example. The picture

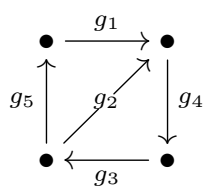

shows an oriented graph with positive weights $g_{1}, \ldots, g_{5}$ on the edges. This graph is connected and without loops. There exists a standard procedure (see, e.g., the Appendix of [5]) to associate two linear spaces with the graph, the linear space of oriented cycles and that of oriented cocycles, and to construct bases in them. 
Electronic Journal of Linear Algebra, ISSN 1081-3810

A cycle is a sequence of edges such that each vertex has as many incoming edges as outgoing ones. A cocycle is a minimal set of edges whose removal disconnects the graph into two subgraphs. In our example, both spaces are subspaces of $\mathbf{R}^{5}$.

To construct bases, one first chooses an arbitrary spanning tree, that is, a minimal subset of the edges that connects all vertices. We take

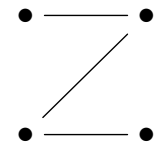

as a spanning tree and denote it by $T$. Removing an edge from $T$ disconnects the vertex set of the graph into two subsets. One of these two subsets contains the outgoing vertex of the removed edge and the other subset contains the the incoming vertex of the edge we removed. The vertices of the former subset are called sources (circles) and the vertices of the latter are referred to as sinks (disks). Then there is a unique cocycle out of the source toward the sink. In our example, removal of $g_{1}, g_{2}, g_{3}$ from $T$ produces the following three linearly independent cocycles:
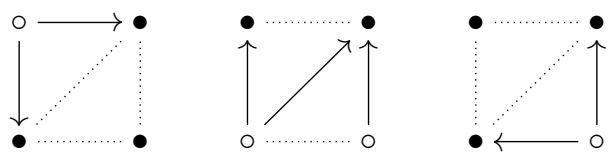

Adding an edge to the spanning tree yields a cycle. This cycle is oriented according to the orientation of the added edge. In our example, we may add the two edges $g_{4}$ and $g_{5}$, and the two resulting cycles

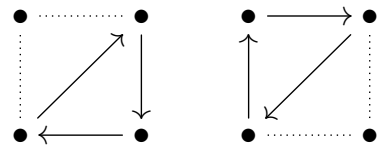

are linearly independent. With the graphs obtained, one then associates columns. Given one of the graphs, the $j$ th entry of the column is 0 if the edge $g_{j}$ is missing in the graph, is 1 if the edge $g_{j}$ is in the graph with the original orientation, and is -1 if the edge $g_{j}$ belongs to the graph with reverse orientation. The five columns we get in our example are

$$
c_{1}=\left[\begin{array}{c}
1 \\
0 \\
0 \\
0 \\
-1
\end{array}\right], \quad c_{2}=\left[\begin{array}{c}
0 \\
1 \\
0 \\
-1 \\
1
\end{array}\right], \quad c_{3}=\left[\begin{array}{c}
0 \\
0 \\
1 \\
-1 \\
0
\end{array}\right], \quad c_{4}=\left[\begin{array}{l}
0 \\
1 \\
1 \\
1 \\
0
\end{array}\right], \quad c_{5}=\left[\begin{array}{c}
1 \\
-1 \\
0 \\
0 \\
1
\end{array}\right] .
$$

The cocycle space is the span of $c_{1}, c_{2}, c_{3}$ and the cycle space is the span of $c_{4}, c_{4}$. 
Let $P \in \mathcal{L}\left(\mathbb{R}^{5}\right)$ be the $5 \times 5$ matrix whose first three rows are $c_{1}^{\top}, c_{2}^{\top}, c_{3}^{\top}$ and whose remaining two rows are zero, and let $Q \in \mathcal{L}\left(\mathbb{R}^{5}\right)$ stand for the $5 \times 5$ matrix whose first three columns are zero and whose last two columns are $c_{4}, c_{5}$,

$$
P=\left[\begin{array}{ccccc}
1 & 0 & 0 & 0 & -1 \\
0 & 1 & 0 & -1 & 1 \\
0 & 0 & 1 & -1 & 0 \\
0 & 0 & 0 & 0 & 0 \\
0 & 0 & 0 & 0 & 0
\end{array}\right], \quad Q=\left[\begin{array}{ccccc}
0 & 0 & 0 & 0 & 1 \\
0 & 0 & 0 & 1 & -1 \\
0 & 0 & 0 & 1 & 0 \\
0 & 0 & 0 & 1 & 0 \\
0 & 0 & 0 & 0 & 1
\end{array}\right]
$$

Obviously, $P$ and $Q$ are complementary projections. In the notation of Section 2, these matrices are given by (2.6) with

$$
\omega=\left[\begin{array}{cc}
0 & -1 \\
-1 & -1 \\
-1 & 0
\end{array}\right] .
$$

Let $G=\operatorname{diag}\left[g_{1}, \ldots, g_{5}\right]$. Straightforward computation gives that

$$
\begin{aligned}
& \operatorname{det}_{+}\left(Q^{*} G Q\right)=\operatorname{det}\left[\begin{array}{cc}
g_{2}+g_{3}+g_{4} & -g_{2} \\
-g_{2} & g_{1}+g_{2}+g_{5}
\end{array}\right], \\
& \operatorname{det}_{+}\left(P G^{-1} P^{*}\right)=\operatorname{det}\left[\begin{array}{ccc}
g_{1}^{-1}+g_{5}^{-1} & -g_{5}^{-1} & 0 \\
-g_{5}^{-1} & g_{2}^{-1}+g_{4}^{-1}+g_{5}^{-1} & g_{4}^{-1} \\
0 & g_{4}^{-1} & g_{3}^{-1}+g_{4}^{-1}
\end{array}\right] .
\end{aligned}
$$

In [4], the matrices on the right are called Kirchhoff-Symanzik matrices. Expanding the determinants we arrive at the following diagrammatic representation:

$$
\begin{aligned}
& \operatorname{det}_{+}\left(Q^{*} G Q\right)=g_{4} g_{5}+g_{1} g_{3}+g_{5} g_{3}+g_{1} g_{4}+g_{2} g_{3}+g_{5} g_{2}+g_{1} g_{2}+g_{4} g_{2}
\end{aligned}
$$

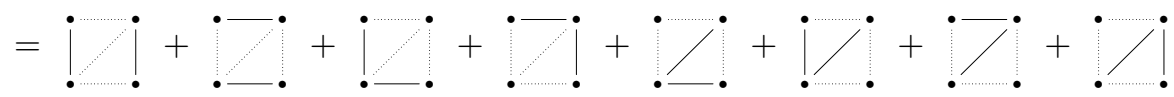

$$
\begin{aligned}
& =\sum_{\mathcal{T}} \prod_{e \notin T} g_{e} \\
& \operatorname{det}_{+}\left(P G^{-1} P^{*}\right)=\frac{1}{g_{1} g_{2} g_{3}}+\frac{1}{g_{2} g_{4} g_{5}}+\frac{1}{g_{1} g_{2} g_{4}}+\frac{1}{g_{2} g_{3} g_{5}}+\frac{1}{g_{1} g_{4} g_{5}}+\frac{1}{g_{1} g_{3} g_{4}}+\frac{1}{g_{3} g_{4} g_{5}}+\frac{1}{g_{1} g_{3} g_{5}}
\end{aligned}
$$

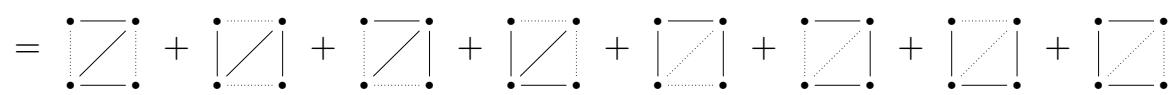

$$
\begin{aligned}
& =\sum_{\mathcal{T}} \prod_{e \in \mathcal{T}} \frac{1}{g_{e}}
\end{aligned}
$$

In the second lines of the expressions we give a representation of the determinant expansion in terms of spanning trees (weights are intended to be multiplied over the solid edges of the diagram), which we compactly resume in the third lines in terms of the spanning-tree polynomials. Replacing in the obvious identity

$$
\left(\prod g_{j}\right) \sum_{\mathcal{T}} \prod_{e \in T} \frac{1}{g_{e}}=\sum_{\mathcal{T}} \prod_{e \notin T} g_{e}
$$

the product and the sums by $\operatorname{det} G, \operatorname{det}_{+}\left(P G^{-1} P^{*}\right), \operatorname{det}_{+}\left(Q^{*} G Q\right)$, we get

$$
\operatorname{det} G \operatorname{det}_{+}\left(P G^{-1} P^{*}\right)=\operatorname{det}_{+}\left(Q^{*} G Q\right),
$$


which is just the second equality in Theorem 1.1. Nakanishi [4, Theorem 3.10] actually proved the equalities

$$
\operatorname{det}_{+}\left(Q^{*} G Q\right)=\sum_{\mathcal{T}} \prod_{e \notin T} g_{e}, \quad \operatorname{det}_{+}\left(P G^{-1} P^{*}\right)=\sum_{\mathcal{T}} \prod_{e \in T} \frac{1}{g_{e}}
$$

in the general case. Thus, Theorem 1.1 for diagonal matrices $G$ and for projections $P$ arising from the above graph theoretic construction is implicitly in the literature at least since 1971. The question whether every skew projection $P$ with entries in $\{0, \pm 1\}$ can be obtained as above, that is, whether it can be represented via a basis of the cocycle space of a graph is more delicate and we do not know the answer. Our contribution here is a simple linear algebra proof of Theorem 1.1, without the journey through graph theory, for arbitrary positive definite $G$, and independently of whether the projection $P$ comes from a cocycle space or not.

The polynomial $T_{\Gamma}(v):=\sum_{\mathcal{T}} \prod_{e \in T} v_{e}$ is referred to as the Tutte polynomial of the graph $\Gamma$. From (3.13) we infer that

$$
T_{\Gamma}(1 / g)=\sum_{\mathcal{T}} \prod_{e \in T} \frac{1}{g_{e}}=\operatorname{det}_{+}\left(P G^{-1} P^{*}\right) .
$$

If $\Gamma$ is a planar graph, there is a well known construction that gives the dual graph $\Gamma^{*}$. Equation (4.11) of [7] says that

$$
T_{\Gamma^{*}}(g)=\operatorname{det} G T_{\Gamma}(1 / g)
$$

Consequently, $T_{\Gamma^{*}}(g)=\operatorname{det} G \operatorname{det}_{+}\left(P G^{-1} P^{*}\right)$, and Theorem 1.1 or (3.12) in combination with (3.13) are equivalent to the equalities

$$
T_{\Gamma^{*}}(g)=\operatorname{det}_{+}\left(Q^{*} G Q\right)=\sum_{\mathcal{T}} \prod_{e \notin T} g_{e}, \quad T_{\Gamma^{*}}(1 / g)=\operatorname{det}_{+}\left(Q^{*} G^{-1} Q\right)=\sum_{\mathcal{T}} \prod_{e \notin T} \frac{1}{g_{e}},
$$

the sums over the set $\mathcal{T}$ of all spanning trees of the original graph $\Gamma$.

We conclude with a beautiful identity that was found in [6] for skew projections emerging in graph theory. This identity reads

$$
P P^{*}+Q^{*} Q=I-\Omega^{2}=\left(I+\Omega^{*}\right)(I+\Omega) \text { for } \Omega=P-P^{*} .
$$

Note that the second equality is obvious because $\Omega$ is skew-symmetric, $\Omega^{*}=-\Omega$. Here are two proofs which show that the identity actually holds for arbitrary complementary projections $P, Q$.

First proof. Using (2.6), we get

$$
P P^{*}+Q^{*} Q=\left(\begin{array}{cc}
I+\omega \omega^{*} & 0 \\
0 & I+\omega^{*} \omega
\end{array}\right), \quad \Omega=P-P^{*}=\left(\begin{array}{cc}
0 & \omega \\
-\omega^{*} & 0
\end{array}\right), \quad \Omega^{2}=\left(\begin{array}{cc}
-\omega \omega^{*} & 0 \\
0 & -\omega^{*} \omega
\end{array}\right),
$$

whence $P P^{*}+Q^{*} Q=I-\Omega^{2}$.

Second proof. Since $P+Q=I=P^{*}+Q^{*}$, we get $P-P^{*}=Q^{*}-Q=\Omega$, it follows that

$$
\begin{aligned}
& \left(P-P^{*}\right)^{2}=P+P^{*}-P P^{*}-P^{*} P=\Omega^{2}, \\
& \left(Q^{*}-Q\right)^{2}=Q+Q^{*}-Q^{*} Q-Q Q^{*}=\Omega^{2},
\end{aligned}
$$

and adding these two equalities, we arrive at

$$
\left(P P^{*}+Q^{*} Q\right)+\left(P^{*} P+Q Q^{*}\right)=2\left(I-\Omega^{2}\right) .
$$


It remains to show that $P P^{*}+Q^{*} Q=P^{*} P+Q Q^{*}$. But this true because

$$
\begin{aligned}
& P P^{*}+Q^{*} Q=P P^{*}+\left(I-P^{*}\right)(I-P)=P P^{*}+I-P^{*}-P+P^{*} P, \\
& P^{*} P+Q Q^{*}=P^{*} P+(I-P)\left(I-P^{*}\right)=P^{*} P+I-P-P^{*}+P P^{*},
\end{aligned}
$$

which completes the proof.

Acknowledgments. We thank the referees for their useful comments. We are especially grateful to one of the referees for notifying us of the argument presented after Theorem 1.3 and leading to (1.2). The research of M. Polettini was supported by the National Research Fund Luxembourg (core project THERMOCOMP) and by the European Research Council (ERC-2015-CoG Agreement No. 681456).

\section{REFERENCES}

[1] A. Böttcher and I.M. Spitkovsky. A gentle guide to the basics of two projections theory. Linear Algebra Appl., 432:14121459,2010

[2] D.Ž. Doković. Unitary similarity of projectors. Aequationes Math., 42:220-224, 1991.

[3] I. Feldman, N. Krupnik, and A. Markus. On the norm of polynomials of two adjoint projections. Integral Equations Operator Theory, 14:69-91, 1991.

[4] S. Nakanishi. Graph Theory and Feynman Integrals. Gordon and Breach, New York, 1971.

[5] M. Polettini. Cycle/cocycle oblique projections on oriented graphs. Lett. Math. Phys., 105:89-107, 2015.

[6] M. Polettini. Oblique projections on metric spaces. Preprint, arXiv:1711.04672, 2020, and arXiv:1711.04672v1, 2017.

[7] A. Sokal. The multivariate Tutte polynomial (alias Potts model) for graphs and matroids. In: B.S. Webb (editor), Surveys in Combinatorics 2005, London Math. Soc. Lecture Note Ser., Cambridge Univ. Press, Cambridge, 327:173-226, 2005.

[8] E. Spigel. On the norm of polynomials of two adjoint projections in Hilbert spaces. Integral Equations Operator Theory, 22:232-241, 1995.

[9] D.B. Szyld. The many proofs of an identity on the norm of oblique projections. Numer. Algorithms, 42:309-323, 2006. 